\title{
Long-ranged dynamic magnetic interaction through low-dimensional systems
}

\author{
A. T. Costa and R. B. Muniz \\ Instituto de Física, Universidade Federal Fluminense, 24210-346 Niterói, RJ, Brazil \\ M. S. Ferreira \\ School of Physics, Trinity College Dublin, Dublin 2, Republic of Ireland \\ D. L. Mills \\ Department of Physics and Astronomy, University of California, Irvine, California 92697, USA
}

(Received 23 October 2008; published 2 December 2008)

\begin{abstract}
Magnetic units attached to low-dimensional metallic nanowires are known to interact via the conduction electrons of the wire leading to preferential alignment directions of the respective magnetic moments. In addition to this so-called static interaction, here we show that a very long-ranged interaction arises when these units are allowed to precess. This dynamic version of the magnetic interaction governs the spin dynamics of the magnetic moments. We are able to relate this interaction to the spin excitation spectrum of the system in which we establish a clear correspondence between the precession relaxation time and the features of the spin susceptibility. We show that the relaxation time is predominantly determined by the availability of noncoherent Stoner modes, a process analogous to Landau damping of plasmons in the electron gas. Contrary to expectations, our results indicate that the range of this interaction is robust against the introduction of disorder. Furthermore, we show that the interaction range is frequency dependent suggesting that it may be tunable by an adequate choice of excitation frequencies. We argue that this may pave the way to controlling the communication between distant parts of a spintronic device.
\end{abstract}

DOI: 10.1103/PhysRevB.78.214403

PACS number(s): 75.30.Ds, 75.30.Et, 75.30.Hx

Magnetic moments embedded in nonmagnetic metallic media interact with each other via the spin polarization induced in the latter. Due to its relevance from fundamental and applied viewpoints, this interaction has been thoroughly investigated during the last two decades ${ }^{1}$ and has proven to play essential roles in recent scientific and technological developments. ${ }^{2}$ Less well known are the dynamical aspects of that coupling: clearly, if one of the magnetizations is set in motion the others will also precess. If the dynamic interaction that produces such induced precession is sufficiently long ranged, this phenomenon may be exploited as a communication channel between distant parts of spintronic devices. When compared to the charge channel traditionally used in electronic devices, the spin channel has the advantage of being almost dissipationless for appropriate choices of materials. Schemes for synchronizing large sets of oscillators have also been devised based on the dynamic coupling between magnetic moments. 3,4

The dynamic coupling is intimately related to the spin current emitted by a magnetic volume whose magnetization has been driven out of equilibrium. ${ }^{5}$ This same spin current is responsible for the enhanced Gilbert damping predicted and observed in a variety of metallic systems ${ }^{6-8}$ and is also closely related to the system's spin excitation spectrum. The dynamic coupling has been investigated in the context of three-dimensional magnetic multilayers, within a semiclassical approach ${ }^{9}$ and a full quantum theoretic analysis. ${ }^{5}$ There, as it has been shown, ${ }^{9}$ the range of the dynamic coupling is limited only by spin-orbit interaction. In that work, however, the frequency dependence of the dynamic coupling was not explored (the frequency of the driving field was assumed to satisfy the condition for ferromagnetic resonance). Furthermore, the motion of the magnetizations was considered within an adiabatic approximation. As far as longwavelength modes are considered, disorder within the magnetic layers should not play a significant role and was not considered in the aforementioned work. However, as we shall see, breaking the translational symmetry in the direction perpendicular to the spin current flow in lowdimensional systems may have important consequences for its behavior.

One of the issues on the subject of spin dynamics that remains open is the physical mechanism that drives the spin precessional motion to relax. In particular, it is important to understand the microscopic origin for this relaxation in the absence of dissipative terms such as spin-orbit coupling known as the primary source of damping for the precessional motion of magnetic moments. Only with a full understanding of the microscopic origin for this relaxation can one establish the relationship of the damping process with the electronic structure of the material in question. However, the vast majority of studies on this topic are essentially based on semiclassical approaches and are insufficient to substantiate this relationship. Our paper is a full quantum-mechanical approach whose goal is to show that the origin of the spin precession damping lies in the availability of incoherent spin excitation modes. These modes are known to be related to the electronic structure of the materials thus providing the microscopic origin for the finite lifetime of spin excitations in low-dimensional systems. With this goal in mind, in this paper we report on theoretical investigations of the dynamical coupling between nanoscopic magnetic volumes mediated by a nearly one-dimensional nonmagnetic metallic system. Furthermore, we present the connection with the spin excitation of the system, highlighting features of the coupling that are not observed in three-dimensional systems. 
To understand the properties of the dynamic coupling it is useful to form a physical picture of the phenomenon. Whenever the magnetization of a magnetic volume (let us call it volume A) coupled to a metallic substrate is driven out of equilibrium, a flow of spin angular momentum is established from the magnetic volume into the substrate. An immediate consequence is that the precession of the magnetization in volume $A$ is damped. ${ }^{5,6,10}$ If there is another magnetic volume (let us call it volume $B$ ) coupled to the nonmagnetic metallic substrate it will absorb part of the spin current emitted by $A$; this will induce a precession of the magnetization $\vec{m}_{B}$ of volume $B$. This precession of $\vec{m}_{B}$ induced by a driving field at $A$ is what we call dynamic coupling between $A$ and $B$. Put it this way; it is evident that the dynamic coupling can be measured as a nonlocal response function, which is also intimately related to the system's excitation spectra. Thus, a measure of the dynamic coupling between two magnetic entities $A$ and $B$ may be defined by the amplitude of precession induced in $B$ by a harmonic circularly polarized transverse field applied locally to $A$. This information is contained in the transverse dynamic susceptibility $\chi_{B A}^{+-}(\omega)$ defined as the Fourier transform of the response function,

$$
\chi_{B A}^{+-}(t)=-i \theta(t)\left\langle\left[S_{B}^{+}(t), S_{A}^{-}(0)\right]\right\rangle .
$$

Here, $S_{A}^{+}$is the spin raising operator at volume $A$ defined as a sum of spin raising operators over all atomic sites that comprise volume $A, S_{A}^{+}=\Sigma_{l \in A} a_{l \uparrow}^{\dagger} a_{l \downarrow}$. Here $a\left(a^{\dagger}\right)_{l \sigma}$ are the annihilation (creation) operators for states localized at site $l$ with spin $\sigma, \theta(t)$ is the Heaviside step function, and $\langle\cdot\rangle$ denotes the expectation value. In this paper, volumes $A$ and $B$ will be collections of magnetic atoms contained in two rings belonging to an infinite cylindrical nanowire.

We consider as a model system a semi-infinite square lattice stripe of side $L$ atoms, with periodic boundary conditions in the transverse direction (mimicking a cylindrical surface). ${ }^{11}$ Because we are interested in investigating the effect of disorder on the dynamic coupling, it is instructive to start with magnetic objects that do not break the translational symmetry of the nanowire along the circumferential direction. In this case we consider two lines containing magnetic atoms separated by a distance $D=N a$, where $a$ is the atomic spacing, as shown schematically in Fig. 1. We want to determine how the dynamic coupling depends on the distance $D$ between magnetic lines and on the atomic composition of the magnetic lines. We call the configuration of a magnetic line the number and distribution of magnetic atoms in it. The configuration of the magnetic line is important in determining the Fourier amplitudes that form the lowest-lying precession mode.

The system's electronic structure is modeled by a singleorbital tight-binding Hamiltonian with effective intra-atomic Coulomb interaction in the magnetic atoms,

$$
H=\sum_{i j} \sum_{\sigma}\left(\epsilon_{i} \delta_{i j}+t_{i j}\right) a_{i \sigma}^{\dagger} a_{j \sigma}+\sum_{i} U_{i} n_{i \uparrow} n_{i \downarrow},
$$

where $i$ and $j$ are the site indices, $\sigma$ is a spin index, $t_{i j}=t$ is the hopping integrals for $i$ and $j$ nearest neighbors and zero otherwise, $\epsilon_{i}$ are the on-site energies, and $U_{i}$ is the effective intra-atomic Coulomb interaction. All energies are measured
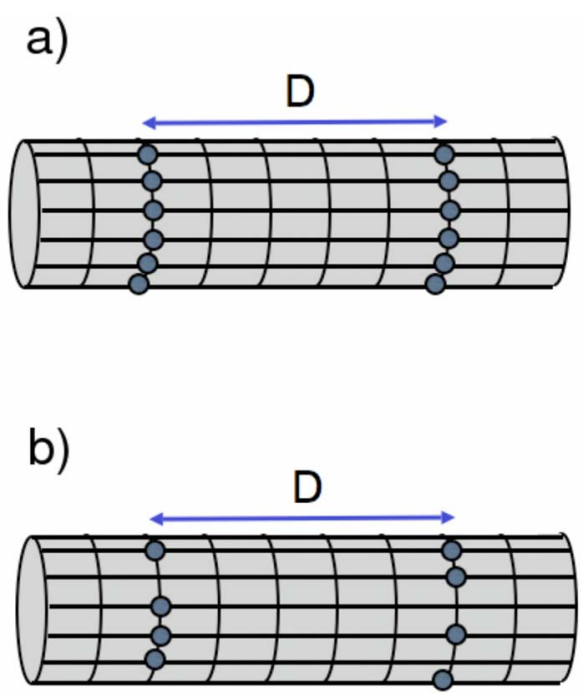

FIG. 1. (Color online) Schematic representations of a section of the infinite metallic cylindrical nanowire with two lines containing magnetic atoms (shown as circles); (a) complete (ordered) magnetic lines and (b) disordered magnetic lines. Nonmagnetic atoms occupy the vertices of the underlying lattice.

in units of the hopping parameter $t$. The magnetic ground state is generated by a mean-field self-consistent approach. Spin excitations are obtained by calculating the transverse susceptibility $\chi_{i j}^{+-}(\Omega)$ within the random-phase approximation, where $i$ and $j$ represent the atomic sites or collections of atomic sites. A static Zeeman field is applied to the system along the $z$ direction in order to stabilize the equilibrium magnetization direction. The value of the Zeeman field is arbitrarily chosen such that a free electron precessing in it would have an energy $\Omega=0.01 t$.

A typical excitation spectrum is shown in Fig. 2, where we plot $-\operatorname{Im} \chi_{A A}^{+-}(\Omega)$ (dashed curve) for two complete magnetic lines embedded in a nonmagnetic wire. Notice that this is not the quantity we use as a measure of the dynamical coupling. Two peaks are clearly visible, corresponding to acoustic (lower energy, in phase) and optical (higher energy,

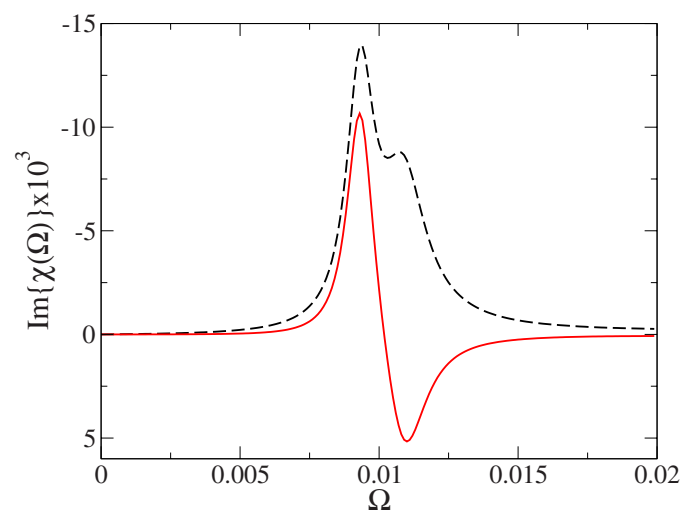

FIG. 2. (Color online) Typical excitation spectrum for two identical magnetic lines separated by $N=50$ atomic spacings. Dashed curve depicts the excitation spectrum projected on one of the magnetic lines; solid curve is the measure of dynamical coupling between lines A and B as a function of driving frequency $\Omega$. 

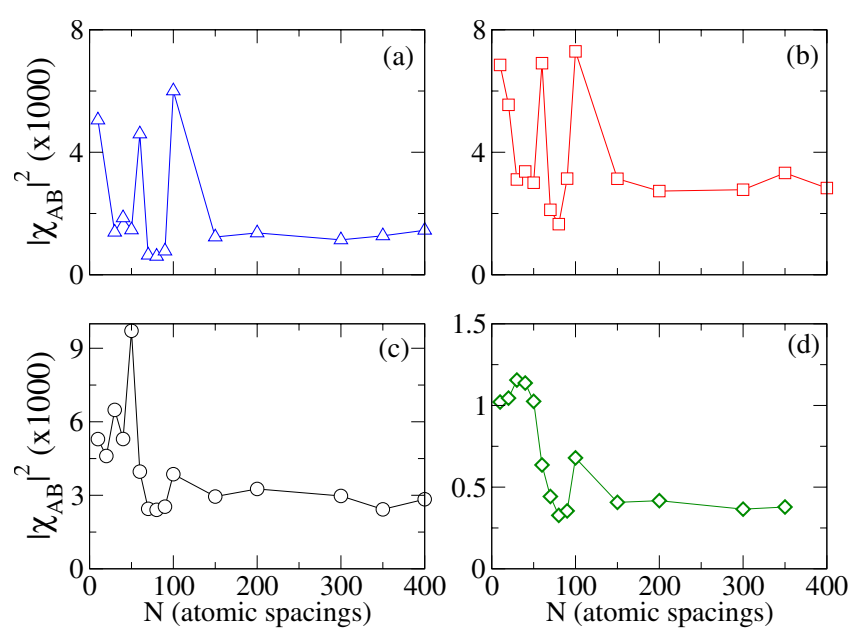

FIG. 3. (Color online) Dynamical coupling between two magnetic rings embedded in a nonmagnetic metallic nanowire as a function of the distance $N$ between them for different frequencies of the driving field: (a) $\Omega=0.008$, (b) $\Omega=0.009 t$, (c) $\Omega=0.010 t$, and (d) $\Omega=0.011 t$.

out of phase) precession modes of $\vec{m}_{A}$ and $\vec{m}_{B}$. We will call the energies of the acoustic and optical mode as $\Omega_{0}$ and $\Omega_{1}$, respectively. The existence of a frequency splitting between the two modes means that there is a static Ruderman-KittelKasuya-Yoshida (RKKY)-type exchange coupling between $\vec{m}_{A}$ and $\vec{m}_{B}{ }^{12}$ which is proportional to the splitting. The solid curve displays a measure of the dynamical coupling, $-\operatorname{Im} \chi_{B A}(\Omega)$; its absolute value is proportional to the amplitude of the magnetization precession induced on $B$ by a transverse circularly polarized field applied on $A$. The sign of $-\operatorname{Im} \chi_{A B}(\Omega)$ is related to the phase difference between the precessing $\vec{m}_{B}$ and the field applied on $A$. The so-called acoustic mode, which is excited in ferromagnetic resonance (FMR) experiments, corresponds to a precession of $\vec{m}_{B}$ in phase with the applied field at $A$; thus $-\operatorname{Im} \chi_{A B}(\Omega)$ is positive at the corresponding frequency $\Omega_{0}$. The finite width of the excitation spectrum $-\operatorname{Im} \chi_{A A}^{+-}(\Omega)$ is directly proportional to the rate of damping of $\vec{m}_{A}$ precession.

From this typical spectrum it is already possible to infer that the dynamical coupling should oscillate as a function of the $A B$ distance $N$, at least for sufficiently small values of $N$. The value of $-\operatorname{Im} \chi_{A B}(\Omega)$ for $\Omega \sim \Omega_{0}$ is influenced by the existence of an optical mode, and such influence depends on the splitting between the two modes. The splitting is proportional to the static exchange coupling between $\vec{m}_{A}$ and $\vec{m}_{B}$, which is known to oscillate as a function of $N .{ }^{12-14}$ The static coupling decays, for quasi-one-dimensional systems, asymptotically as $1 / N$; hence oscillations in the dynamic coupling should be seen even for relatively large values of $N$.

We may now inquire about the behavior of $\left|\chi_{A B}(\Omega)\right|^{2}$ as the distance $N$ between rings $A$ and $B$ is varied. It is expected that the spin current that couples dynamically the two magnetizations should suffer the effects of quantum interferences as the $A B$ distance is changed. Figure 3 shows the dynamic coupling as a function of the distance $N$ between rings $A$ and $B$ for a few frequencies of the driving field. For all frequencies quantum oscillations are clearly visible, and the cou-

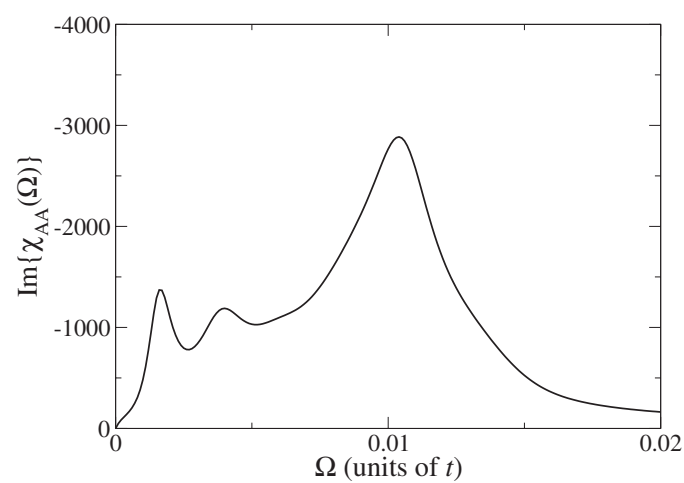

FIG. 4. Excitation spectrum of a tube with two magnetic rings where disorder has been introduced (see text). The distance between the magnetic rings is 20 atomic spacings.

pling reaches a finite asymptotic value for very large $N$. This is in clear contrast with the behavior of the static coupling that, although long ranged, goes to zero as $N \rightarrow \infty$. The spatial modulation of the dynamic coupling may be understood as a property of the system's spin excitations. The spin precession, which generates the spin current responsible for the dynamic coupling, is a collective excitation of the system. It may be resolved in a superposition of spin-wave modes with different wave vectors $Q$ parallel to the rings, which decay into (incoherent) Stoner excitations: a process analogous to Landau damping of plasmons in the electron gas. Only the uniform $(Q=0)$ spin-wave component has infinite lifetime (in the absence of spin-orbit coupling). This component is the origin of the very long range of the dynamic coupling predicted in magnetic multilayers..$^{9}$ If the magnetic region is translationally invariant in the direction perpendicular to the flow of spin current, as is the case in this example, the dominant spin-wave component has $Q=0$. Thus, after the $Q \neq 0$ components have been damped, there remains considerable precession amplitude flowing through the nonmagnetic substrate.

When translation symmetry is broken by, for example, introducing disorder, new modes appear that correspond to superpositions of finite $Q$ modes; an example of such spectrum is shown in Fig. 4. The magnetic volumes are now rings of 20 atoms, each where half the atoms have been randomly replaced by nonmagnetic atoms. It is clear that the spectrum has more structure, meaning that more modes are present with energy comparable to that of the $Q=0$ mode.

It can be seen in Fig. 5 that the dynamic coupling at the frequencies associated with the new $Q \neq 0$ modes decays toward zero as the distance between the magnetic volumes increase. This decaying is, as stated previously, a consequence of coherent spin-wave modes decaying into incoherent Stoner excitations (we remind the reader that there is no spin relaxation in our model). This kind of process can only be properly described in the framework of itinerant magnetism.

Even in the presence of disorder, modes with frequencies close to that of the uniform mode have considerable amplitude in the $Q=0$ component. We illustrate this point in Fig. 5(a). This means that if frequency can be tuned, long-range dynamic coupling can be obtained even in disordered systems. 

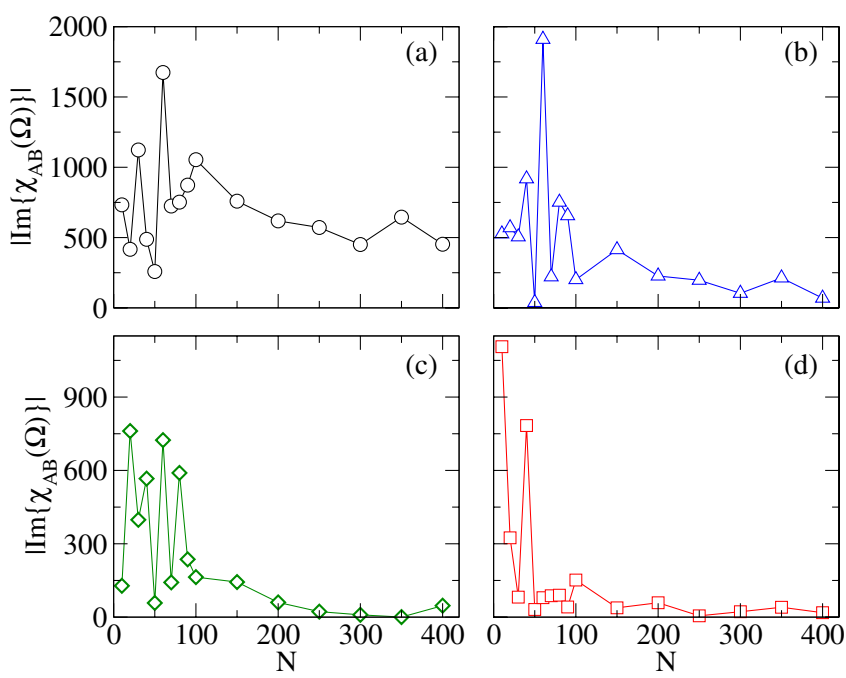

FIG. 5. (Color online) Dynamic coupling as a function of distance $N$ between disordered magnetic volumes $A$ and $B$ for selected mode energies: (a) $\Omega=0.01 t$, (b) $\Omega=0.0056 t$, (c) $\Omega=0.004 t$, and (d) $\Omega=0.014 t$.

From the theoretical point of view $\chi_{A B}$ is the best choice to characterize the dynamic coupling between volumes $A$ and $B$. Experimentally, however, it can be a very difficult quantity to measure. Its direct measurement requires that a driving field is applied to $A$, and then $\vec{m}_{B}$ is measured. In systems of nanoscopic dimensions this kind of measurement requires a spatial resolution not easily available presently. The system's FMR spectrum, given by $\chi=\chi_{A A}+\chi_{A B}+\chi_{B A}+\chi_{B B}$, is an alternative way to measure the dynamic coupling although it provides a slightly different kind of information. The halfwidth at half maximum (HWHM) of $\chi(\Omega)$ is proportional to the damping rate of the FMR mode, $1 / \tau$. The FMR mode corresponds to an in-phase precession of all individual magnetic moments in the system. To see how its lifetime is related to the dynamic coupling it is useful to invoke once again the spin currents emitted by the precessing magnetizations. If only volume $A$ is in contact with the infinite substrate the current it emits flows away from it indefinitely, resulting in a damping rate, $1 / \tau_{0}$. If, however, volume $B$ is also attached to the substrate, part of the current emitted by $A$ is absorbed by $B$ causing $\vec{m}_{B}$ to precess and emit itself a spin current that will be partly absorbed by $A$. The end result of this multiple-scattering process will be a trapping of some of the spin current that would otherwise flow to the infinite substrate. Consequently the lifetime $\tau$ of the FMR mode is increased by the presence of a second magnetic volume. Thus, by comparing $\tau_{0}$ with $\tau$ it is possible to identify the dynamic coupling between $A$ and $B$. For instance, the less strongly coupled the magnetic unities are, the more the FMR mode's lifetime approaches that of a single magnetic unit. The dynamic coupling should lead, as discussed above, to larger lifetimes and thus smaller mode width. In Fig. 6 we present the half-width at half maximum $\Delta \Omega$ of the calculated ferromagnetic resonance spectra of an infinite wire with two complete magnetic rings embedded in it, as the distance $N$ between the rings is varied. We compare those results with the value of $\Delta \Omega_{\text {single }}$ extracted from the FMR spectrum of a

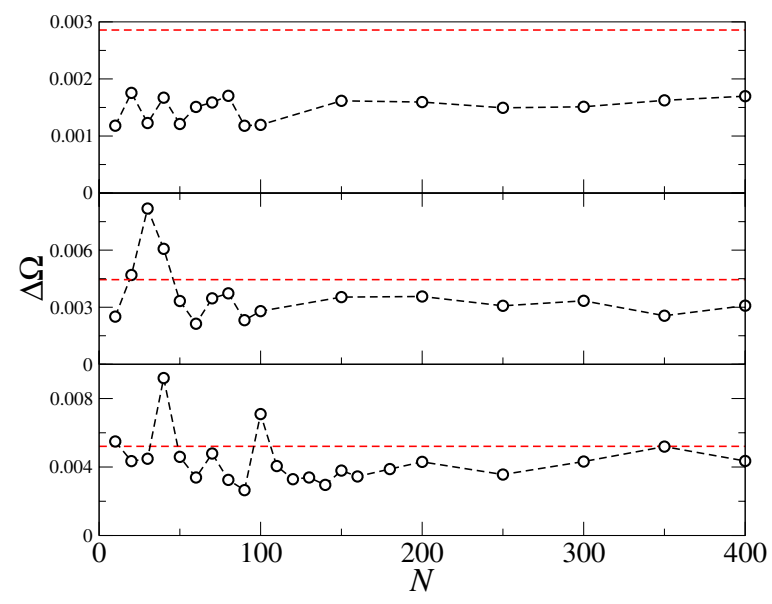

FIG. 6. (Color online) Half-width at half maximum of the FMR spectra for (a) two complete magnetic rings, (b) two magnetic rings with half of the magnetic atoms replaced randomly by nonmagnetic atoms, and (c) two magnetic atoms, all embedded in infinite wires, as functions of the distance between the rings. The red dashed line indicates the HWHM of the FMR spectrum of a single magnetic unity embedded in similar wires.

single complete magnetic ring embedded in a similar wire. It is clear that $\Delta \Omega_{\text {single }}<\Delta \Omega$ for all values of $N$ considered and also that it oscillates around a reasonably constant value. It is interesting to notice that the trend observed in Fig. 6(a) is similar to what is seen in Fig. 3, which corresponds to the dynamic coupling carried by the uniform mode. This confirms that $\Delta \Omega$ is a good measure of the dynamic coupling although it can only provide information about a very narrow frequency interval around the resonance. In Figs. 6(b) and 6(c) we can see the effect of spacial inhomogeneities on the dynamic coupling: in (b) each magnetic ring has ten magnetic atoms and ten nonmagnetic atoms distributed randomly; and in (c) there is only one magnetic atom in each ring. The linewidths are clearly much closer to $\Delta \Omega_{\text {single }}$ in both cases. However, since $\Delta \Omega$ is essentially a property of the $Q=0$ mode and this mode always lead to long-range coupling, neither of them are identical to $\Delta \Omega_{\text {single }}$.

To summarize, we investigated the properties of dynamic coupling between magnetic moments embedded in a lowdimensional metallic substrate. We showed that this coupling is large and long ranged in the absence of disorder. The dynamic coupling displays strong oscillations as a function of the distance between magnetic volumes, a feature not observed in bulk systems. For disordered magnetic volumes there are several frequencies for which the coupling is sizeable but decays toward zero as the distance between magnetic volumes increase. This decaying is due to the presence of Stoner excitations that damp collective modes with nonzero wave vectors. Even in the presence of disorder, however, it is possible to obtain a long-ranged dynamic coupling by choosing the pumping frequency appropriately. We also showed how the dynamic coupling manifests itself in the linewidths of FMR spectra, which are easily accessible experimentally. The diversity of spatial modulations revealed by our calculations suggest that with clever material engineering, it may be possible to control long-distance commu- 
nication in nanoscale devices. The combination of a longranged exchange interaction with a dynamic coupling only limited by spin-flip scattering may lead to unusual excitation properties. Further investigation on this topic is presently underway.
A.T.C. and R.B.M. acknowledge financial support from $\mathrm{CNPq}$ and FAPERJ. M.S.F. acknowledges the financial support from Science Foundation Ireland. D.M.L. acknowledges support from the U.S. Department of Energy through Grant No. FG03-84ER-45083.
${ }^{1}$ M. D. Stiles, J. Magn. Magn. Mater. 200, 322 (1999).

${ }^{2}$ M. N. Baibich, J. M. Broto, A. Fert, F. Nguyen Van Dau, F. Petroff, P. Etienne, G. Creuzet, A. Friederich, and J. Chazelas, Phys. Rev. Lett. 61, 2472 (1988).

${ }^{3}$ S. Kaka, M. Pufall, W. Rippard, T. Silva, S. Russek, and J. Katine, Nature (London) 437, 389 (2005).

${ }^{4}$ J. Grollier, V. Cros, and A. Fert, Phys. Rev. B 73, 060409(R) (2006).

${ }^{5}$ A. T. Costa, R. B. Muniz, and D. L. Mills, Phys. Rev. B 73, 054426 (2006).

${ }^{6}$ L. Berger, Phys. Rev. B 54, 9353 (1996).

${ }^{7}$ J. C. Slonczewski, J. Magn. Magn. Mater. 195, L261 (1999).

${ }^{8}$ K. Lenz, T. Tolinski, J. Lindner, E. Kosubek, and K. Baberschke,
Phys. Rev. B 69, 144422 (2004).

${ }^{9}$ B. Heinrich, Y. Tserkovnyak, G. Woltersdorf, A. Brataas, R. Urban, and G. E. W. Bauer, Phys. Rev. Lett. 90, 187601 (2003).

${ }^{10}$ Y. Tserkovnyak, A. Brataas, G. E. W. Bauer, and B. I. Halperin, Rev. Mod. Phys. 77, 1375 (2005).

${ }^{11}$ A. T. Costa, R. B. Muniz, and M. S. Ferreira, New J. Phys. 10, 063008 (2008).

${ }^{12}$ A. T. Costa, D. F. Kirwan, and M. S. Ferreira, Phys. Rev. B 72, 085402 (2005).

${ }^{13}$ A. T. Costa, C. G. Rocha, and M. S. Ferreira, Phys. Rev. B 76, 085401 (2007).

${ }^{14}$ D. F. Kirwan, C. G. Rocha, A. T. Costa, and M. S. Ferreira, Phys. Rev. B 77, 085432 (2008). 